\title{
ANÁlise DA DISTRIBUIÇÃO DA ISONIAZIDA PARA O ENFRENTAMENTO DA ILTB E TUBERCULOSE NO MUNICÍPIO DO RECIFE
}

\author{
T. E. H. LEÃO ${ }^{1}$, M. N. S. de C. BARRETO ${ }^{2}$, J. M. de ALMEIDA ${ }^{2}$, A.D. de MELO Jr ${ }^{2}$ \\ ${ }^{1}$ Universidade Federal de Pernambuco, Centro de Ciências da Saúde, Departamento de \\ Ciências Farmacêuticas. \\ ${ }^{2}$ Prefeitura Municipal do Recife, Secretaria de Saúde, Gerência Geral de Assistência \\ Farmacêutica.
}

E-mail para contato: tata hleao@hotmail.com

\begin{abstract}
RESUMO - A Isoniazida é o antimicobacteriano utilizado no tratamento da Infecção Latente de Tuberculose e também compõe os esquemas padrões para o tratamento desta patologia. Objetivo: Analisar, através da comparação com os casos de tuberculose notificados no Sistema Nacional de Agravos de Notificação (SINAN) e com as orientações preconizadas pelo Ministério da Saúde (MS), a distribuição da Isoniazida 100mg e sua contribuição para o Controle da Tuberculose. Métodos: Trata-se de um estudo retrospectivo comparativo das orientações preconizadas pelo Ministério da Saúde, com os dados de Notificação compulsória e com o consumo de Isoniazida no período de janeiro de 2013 a dezembro de 2015. Os relatórios foram obtidos do SINAN e do sistema Hórus Recife. Resultados: Em 2013, 2014 e 2015 foram notificados respectivamente, 1610, 1529, 1399 casos novos de tuberculose e a distribuição de Isoniazida $100 \mathrm{mg}$ foram de 120.530, 228.320, e 69.586 comprimidos respectivamente. A diminuição de número de casos novos em 2015 enfatiza a importância da quimioprofilaxia. Conclusão: Os resultados apresentados demonstram consonância da assistência à saúde com os guias e orientações do Ministério da Saúde para o enfrentamento da tuberculose. Além disso, respalda a importância de uma prescrição que segue as diretrizes do Uso Racional de Medicamentos.
\end{abstract}

Palavras Chave: Diretrizes, Endemia, Hórus, Medicamento.

ABSTRACT - Isoniazid is the antimycobacterial used in the treatment of Latent Tuberculosis Infection and also composes standard regimens for the treatment of this pathology. Objective: To analyze the distribution of Isoniazid 100mg and its contribution to the Control of Tuberculosis, through the comparison with tuberculosis cases reported in SINAN and with the guidelines recommended by the Ministry of Health. Methods: This is a retrospective comparative study of the guidelines recommended by the Ministry of Health, with the data of compulsory Notification and consumption of Isoniazid from January 2013 to December 2015. The data were obtained from the National System of Notifiable Diseases (SINAN) and the Horus Recife system. Results: The results presented demonstrate 
compliance of health care with the guidelines and guidelines of the Ministry of Health for the treatment of tuberculosis. In addition, it supports the importance of a prescription that follows the Rational Drug Use guidelines.

Keywords: Guidelines, Endemic, Hórus, Medicine.

\section{INTRODUÇÃO}

A Tuberculose (TB) é uma doença infectocontagiosa com incidência endêmica, provocada pelo Mycobacterium tuberculosis, que afeta prioritariamente os pulmões, pois, é a forma mais atenuada de promover a proliferação da doença. O diagnóstico da TB é dado pela positividade do teste tuberculínico associado à exclusão de tuberculose ativa (BRASIL, 2011; BRASIL, 2017a).

A vulnerabilidade de contrair a infecção é praticamente universal, porém nem sempre esta doença se manifesta devido ao desenvolvimento de imunidade parcial. No estado latente, os bacilos permanecem encapsulados, nem progridem e nem provocam o adoecimento. Esta é a infecção latente da tuberculose (ILTB), que se expressa, na maioria das vezes, pela prova tuberculínica ou teste IGRA positivo (BRASIL, 2017; SIQUEIRA, MENDONÇA, PENEDO, 2012).

Segundo a Organização Mundial de Saúde em 2015, 10,4 milhões de pessoas adoeceram com a TB, dentre elas 1,8 chegaram a óbito. Neste mesmo ano 69 mil pessoas adoeceram no Brasil, dentre elas 4,5 chegaram a óbito (BRASIL, 2017b). Já no município de Recife nos anos de 2012 e 2013, foram diagnosticados, respectivamente, 1.575 e 1.538 casos novos de tuberculose, apresentando taxas de detecção de 100,4 e 98,0 casos por 100.000 habitantes. (RECIFE, 2014)

O tratamento de escolha para a TB no Brasil se dá com associações de medicamentos, utilizando esquemas padrões preconizado. A Isoniazida é a mais utilizada por ser juntamente com a Rifampicina a mais potente das drogas, pois são ativas em todas as populações bacilares sensíveis. Além disso, a Isoniazida é utilizada para o tratamento de ILTB como profilaxia, recomendada pela Organização Mundial da Saúde (OMS) (SIQUEIRA, MENDONÇA, PENEDO, 2012).

O objetivo deste trabalho foi realizar um estudo retrospectivo, analisando através da comparação com os casos de tuberculose notificados no SINAN e com as orientações preconizadas pelo Ministério da Saúde, a distribuição da Isoniazida 100mg e sua contribuição para o Controle da Tuberculose.

\section{MATERIAIS E MÉTODOS}

Trata-se de um estudo descritivo com análise retrospectiva da distribuição da Isoniazida no município de Recife e casos novos de TB no período de três anos. Os relatórios foram obtidos pelo Sistema Nacional de Agravos de Notificação (SINAN) e do sistema Hórus Recife, no período de janeiro de 2013 a dezembro de 2015. Para análise comparativa utilizou-se os Manuais de Recomendação para controle de Tuberculose e 
outros documentos produzidos pelo Ministério da Saúde, além dos dados epidemiológicos para comparar a distribuição da Isoniazida.

\section{RESULTADOS E DISCUSSÃO}

Pressupõe-se que 50 milhões de brasileiros estejam infectados por Mycobacterium tuberculosis, nos quais 5\% destes desenvolverão a doença. O restante dos $95 \%$ expostos irá desenvolver uma resposta imune efetiva, a ILTB (LOPES, PINHEIRO, MONTEIRO, QUEIROZ, MADEIRA, LOPES, 2011). Podemos observar na Tabela 1 a seguir o número de casos novos de TB registrados, frente ao número de comprimidos de Isoniazida distribuídos no município do Recife.

Tabela 1. Número de casos novos de tuberculose em comparação com o número de comprimidos de Isoniazida distribuídos no período de 2013 a 2015 no município do Recife.

\begin{tabular}{rcc}
\hline Período do Estudo & Casos Novos & $\begin{array}{c}\mathbf{N}^{0} \text { de comprimidos de } \\
\text { Isoniazida distribuídos }\end{array}$ \\
\hline $\mathbf{2 0 1 3}$ & 1.610 & 120.530 \\
$\mathbf{2 0 1 4}$ & 1.529 & 228.320 \\
$\mathbf{2 0 1 5}$ & 1.399 & 69.586 \\
\hline
\end{tabular}

De acordo com a Tabela 1, percebe-se um incremento na distribuição de Isoniazida 100mg em 2014 e uma diminuição dos casos novos em 2015, enfatizando a importância da quimioprofilaxia. Os dados apresentados confirmam o que preconiza o Manual de recomendação para o controle de Tuberculose, em que o principal uso da Isoniazida é para o tratamento de ILTB, apesar do risco de hepatotoxicidade relacionado ao seu uso.

Obtemos também a confirmação do que se determina no Guia de Vigilância em Saúde (Vol. 2), onde indica o uso da Isoniazida no tratamento da ILTB, reduzindo o risco de adoecimento por TB ativa em $60 \%$ a 90\%, no qual esta variação se deve à duração e a adesão ao tratamento (BRASIL, 2017a). O Plano Nacional pelo Fim da Tuberculose como Problema de Saúde Pública tem como meta implantar esquemas encurtados de tratamento da ILTB com o objetivo de melhorar a adesão a essa estratégia pelo fim da TB, isso vem demonstrado na variação dos anos 2014 a 2015 (BRASIL, 2017b).

\section{CONCLUSÃO}

O crescimento do tratamento para ILTB se deve as várias ações em saúde que ao longo desses últimos anos tem sido desenvolvido na cidade do Recife, junto aos profissionais para o enfrentamento da TB com priorização de alguns tópicos como o exame dos contatos e diagnostico da ILTB. O uso maior de Isoniazida para os casos de ILTB demonstra uma consonância da assistência à saúde com os guias e orientações do Ministério da Saúde para o enfrentamento da tuberculose e a sua erradicação. 


\section{REFERÊNCIAS}

Brasil. Ministério da Saúde. Secretaria de Vigilância em Saúde. Coordenação-Geral de Desenvolvimento da Epidemiologia em Serviços. Guia de Vigilância em Saúde : volume 2 / Ministério da Saúde, Secretaria de Vigilância em Saúde, Coordenação-Geral de Desenvolvimento da Epidemiologia em Serviços. - 1. ed. atual. - Brasília : Ministério da Saúde, 2017a.

Brasil. Ministério da Saúde. Secretaria de Vigilância em Saúde. Departamento de Vigilância das Doenças Transmissíveis. Brasil Livre da Tuberculose : Plano Nacional pelo Fim da Tuberculose como Problema de Saúde Pública / Ministério da Saúde, Secretaria de Vigilância em Saúde, Departamento de Vigilância das Doenças Transmissíveis. - Brasília : Ministério da Saúde, 2017b.

RECIFE, Governo Municipal, Secretaria de Saúde do Recife, Secretaria Executiva de Coordenação Geral, Gerência Geral de Planejamento. Plano Municipal de Saúde 2014 2017 / Governo Municipal, Secretaria de Saúde do Recife, Recife. Secretaria Executiva de Coordenação Geral, Gerência Geral de Planejamento. _ 1 ${ }^{\mathrm{a}}$. Ed. - Secretaria de Saúde do Recife, 2014.

Lopes DMA, Pinheiro VGF, Monteiro HSA, Queiroz JAN, Madeira LS, Lopes MMA. Diagnóstico e tratamento da tuberculose latente em pacientes com doenças inflamatórias crônicas e uso de imunobiológicos inibidores do TNF- $\alpha$. J Bras Pneumol. 2011;37(3):308-316

Penedo CC, Siqueira KZ e Mendonça SA. Indicação da prova tuberculínica e infecção latente da tuberculose em HIV-positivos, Município de Blumenau, Estado de Santa Catarina, Brasil, 2004-2009. Epidemiol. Serv. Saúde, Brasília, 21(4):635-644, out-dez 2012.

\section{AGRADECIMENTOS}

Agradeço a Secretaria de Saúde do Recife, a equipe da Coordenação de Tuberculose e da Gerência de Assistência Farmacêutica, pelo fornecimento dos dados para realização da pesquisa. 